\section{Hyperopic refractive errors as a prognostic factor in intermittent exotropia surgery}

MK Kim, US Kim, M-J Cho and S-H Baek

\begin{abstract}
Purpose To evaluate and compare surgical outcomes with respect to refractive errors in strabismus surgery for the treatment of intermittent exotropia (IXT).

Methods The medical records of patients with IXT who were treated by one surgeon from January 2005 and June 2011 were reviewed. Three hundred and thirty-three IXT patients were included and divided into three groups according to preoperative refractive error: IXT with hyperopia (group I), IXT with emmetropia (group II), and IXT with myopia (group III). The surgical outcomes with respect to sensory and motor criteria were compared among the three groups.

Results The surgical success rates according to motor criteria and sensory and motor criteria combined were higher in groups I (29 patients) and III (124 patients) than in group II (180 patients) at postoperative 3 and 6 months and at the last follow-up. Stereopsis was significantly better in groups II and III than in group I preoperatively $(P=0.002$ by one-way analysis of variance test); however, the difference was not significant postoperatively. Twenty patients in group I $(69.0 \%)$ were prescribed undercorrected hyperopic spectacles postoperatively, while only 22 patients in group III (17.7\%) were prescribed spectacles with more myopic power than their refractive errors.

Conclusion In the surgical treatment of IXT, hyperopia was not an indicator of poor prognosis. Taking into consideration the age effect, follow-up period after IXT surgery, and stereopsis improvement, hyperopic refractive error is rather a good prognostic factor. Eye (2015) 29, 1555-1560; doi:10.1038/eye.2015.152; published online 21 August 2015
\end{abstract}

\section{Introduction}

Intermittent exotropia (IXT) is one of the most common types of strabismus, especially in
Asian patients. ${ }^{1-3}$ Although it is a widely studied disease, there are many unanswered questions regarding best management practices and prognosis. Controversies partly stem from the highly variable surgical success rate in the published literature, ranging from 42 to $81 \%{ }^{4-9}$ To evaluate the success of IXT surgery, functional outcomes are emphasized, ${ }^{5-7,10-19}$ as well as motor alignment after surgery; however, few studies have considered functional outcomes for evaluating surgical success. 5, 7,10 Several studies have investigated factors that possibly affect surgical outcomes, including refractive errors. The effects of refractive errors in the management of IXT are reported as variable, especially with respect to hyperopia. Generally, hyperopic correction can decrease the demand on the accommodative convergence and thus increase exodeviation. ${ }^{20}$ However, some cases reported a paradoxical decrease in exodeviation after hyperopic correction for improved fusional convergence. $^{21,22}$ In this study, we reviewed the records of patients who underwent strabismus surgery for IXT to evaluate and compare surgical outcomes considering refractive errors by motor and sensory criteria for surgical success rates.

\section{Materials and methods}

We conducted a retrospective review of the medical records of subjects who underwent surgery for IXT by one surgeon (S-HB) at Kim's Eye Hospital between January 2005 and June 2011, with $>6$ months of postoperative follow-up. This study was approved by the Institutional Review Board of the Kim's Eye Hospital, Seoul, Korea.

The following preoperative characteristics were recorded from the patients' charts: age at onset, age at surgery, best-corrected visual acuity, cycloplegic refraction, preoperative motor alignment at distance and near, and
Department of

Ophthalmology, Kim's Eye Hospital, Myung-Gok Eye

Research Institute, Konyang University College of Medicine, Seoul, Korea

Correspondence: S-H Baek, Department of Ophthalmology, Kim's Eye Hospital, Myung-Gok Eye Research Institute, Konyang University College of Medicine, Youngdeungpo 4th 156, Youngdeungpo-gu, Seoul 150-034, Korea Tel: +82 226397811 ; Fax: +82 226333976 E-mail: drslitlamp@ kimeye.com

Received: 8 May 2015 Accepted: 24 June 2015 Published online: 21 August 2015

Presented at the 2013 American Association for Pediatric Ophthalmology and Strabismus-Singapore National Eye Centre Joint Meeting, Singapore, 14-16 July 2013 
stereoacuity. The angle of deviation was determined by the prism alternate cover technique with an accommodative target at both distance $(6 \mathrm{~m})$ and near $(1 / 3 \mathrm{~m})$. Sensory testing was performed using the Titmus tests. Refractive errors were analyzed by spherical equivalent values. The difference of $>1$ dioptre (D) in spherical equivalent between the refractive errors of each eye was considered as anisometropia. Patients with a difference of two lines or more of visual acuity in each eye, or with visual acuity of each eye $<20 / 30$, were considered to have amblyopia. Patients with constant exotropia, oblique muscle dysfunction, vertical strabismus, A-V pattern strabismus, paralytic strabismus, previous extraocular muscle surgery, severe amblyopia, anisometropia, nystagmus, and neurological or other medical problems were excluded from this study.

Patients were classified as having basic IXT if the difference between distant and near deviation was within 10 prism dioptres (PDs). Convergence insufficiency type was defined as an IXT at near measurement at least 10 PDs greater than at distance. If the distant angle of exodeviation was $>10$ PD larger than the near angle of exodeviation, the angle of deviation was re-measured after $>1 \mathrm{~h}$ of monocular occlusion of the non-dominant eye to discriminate between pseudo-divergence excess and divergence excess.

Patients were divided into three groups according to preoperative refractive error: patients with spherical equivalent in both eyes $\geq+1 \mathrm{D}$ were classified as group I (hyperopic IXT group), patients with spherical equivalent of both eyes between $-1 \mathrm{D}$ and $+1 \mathrm{D}$ were classified as group II (emmetropic IXT group), and patients with spherical equivalent of both eyes $\leq-1 \mathrm{D}$ were classified as group III (myopic IXT group). The result was considered successful when the distant deviation with correction was $\leq 5$ PD esodeviation or $<15$ PD exodeviation by motor criteria, and when postoperative stereopsis was $\leq 100$ arcsec with sensory criteria. Reoperation was considered a failure and cases that received reoperation were included in this study until the last follow-up visit before their reoperation. The surgical success rates were evaluated at 3, 6, and 12 months after surgery and at the last follow-up according to motor and functional criteria success, and these were compared between the three groups.

Glasses were prescribed before surgery whenever needed, ie, when there were significant myopic, hyperopic, and/or astigmatic refractive errors. The target angle for the surgery was the total exotropic angle obtained with maximum tolerated hyperopic correction or with full myopic and astigmatic correction. Patients were prescribed undercorrected hyperopic spectacles and spectacles more myopic than their own refractive errors postoperatively when they appeared to show increasing exodeviation.
For the statistical analysis, one-way analysis of variance (ANOVA), Chi-square test, and repeated measures ANOVA were used, with a significance level of $P<0.05$ without Bonferroni correction or reduced values with Bonferroni correction when needed. All statistical data were analyzed using the PASW software for Windows, version 18.0 (IBM Corp., Armonk, NY, USA).

\section{Results}

A total of 333 patients were included, and among them, 29 were sorted into group I, 180 to group II, and 124 to group III. Comparisons of baseline characteristics between the three groups are presented in Table 1. There were statistically significant differences in the ages and preoperative stereopsis of the groups; patients in group III were significantly older than the other patients $(P=0.001$ by one-way ANOVA test), and preoperative stereopsis was significantly worse in group I than in the other groups ( $P=0.002$ by one-way ANOVA test). The follow-up period seemed to be longer in group I, but statistical significance was not reached. Other factors, including sex, time from diagnosis to surgery, preoperative angle of deviation, type of exotropia, and type of surgery, were not statistically different between the three groups.

The surgical success rate according to motor criteria only was higher in group I and III than in group II at postoperative 3, 6, and 12 months and at the last follow-up. At the last follow-up, there was a statistically significant difference in the success rate according to group ( $P=0.001$ by Chi-square test); however, comparisons between group I (62.1\%) and group III $(72.1 \%)$ demonstrated no significant difference $(P=0.26$ by Chi-square test, Figure 1).

There was a significant difference in preoperative and postoperative stereopsis between the three groups ( $P=0.007$ by repeated-measures ANOVA). By post-hoc comparison with a significant $P$-value $<0.01$ with Bonferroni correction, stereopsis was significantly better in groups II and III than in group I preoperatively ( $P=0.002$ by one-way ANOVA test); however, the difference was not significant postoperatively (Table 2).

The surgical success rate considering motor and sensory criteria together was also higher in groups I and III than in group II at postoperative 3 and 6 months and at the last follow-up. Again, there was a statistically significant difference in the success rates between the three groups at the last follow-up $(P=0.004$ by Chi-square test); however, the difference between group I (61.5\%) and group III (66.1\%) was not significant $(P=0.066$ by Chi-square test, Figure 2).

After surgery, glasses were manipulated usually when the patients showed a tendency to exodrift. Twenty patients in group I $(69.0 \%)$ were prescribed 
Table 1 Baseline clinical characteristics of the patients stratified by preoperative refractive errors

\begin{tabular}{|c|c|c|c|c|}
\hline Factor & Group I $(\mathrm{n}=29)$ & Group II $(\mathrm{n}=180)$ & Group III $(\mathrm{n}=124)$ & P-value \\
\hline Age at surgery (years) & $7.02 \pm 4.48$ & $7.73 \pm 3.94$ & $10.47 \pm 6.35$ & $<0.001^{*}$ \\
\hline Sex (M:F) & 19: 10 & 98: 82 & 63: 61 & $0.355^{+}$ \\
\hline Age at diagnosis (years) & $5.56 \pm 4.89$ & $6.51 \pm 4.29$ & $9.51 \pm 6.58$ & $<0.001^{*}$ \\
\hline Time from diagnosis to surgery (years) & $1.45 \pm 1.43$ & $1.22 \pm 1.46$ & $0.95 \pm 1.68$ & $0.170^{*}$ \\
\hline Preoperative distant deviation (PD) & $27.03 \pm 6.20$ & $26.85 \pm 6.14$ & $26.54 \pm 7.86$ & $0.905^{*}$ \\
\hline Preoperative near deviation (PD) & $26.93 \pm 7.69$ & $25.88 \pm 8.89$ & $26.89 \pm 9.37$ & $0.588^{*}$ \\
\hline Preop stereopsis (log arcsec) & $2.29 \pm 0.58$ & $2.03 \pm 0.41$ & $1.92 \pm 0.34$ & $0.002^{*}$ \\
\hline Type of exotropia & & & & $0.128^{+}$ \\
\hline Basic & 22 & 153 & 107 & Total $=282$ \\
\hline Convergence insufficiency & 3 & 5 & 9 & Total $=17$ \\
\hline Divergence excess & 0 & 5 & 3 & Total $=8$ \\
\hline Pseudodivergence excess & 4 & 17 & 5 & Total $=26$ \\
\hline Follow-up period after surgery (months) & $35.24 \pm 26.80$ & $26.94 \pm 19.61$ & $26.03 \pm 19.53$ & $0.084^{*}$ \\
\hline Immediate preoperative cycloplegic refraction (D) & $2.05 \pm 1.47$ & $0.07 \pm 0.44$ & $-2.39 \pm 1.42$ & $<0.001^{*}$ \\
\hline First surgery type & & & & $0.309^{+}$ \\
\hline $\mathrm{R}$ and $\mathrm{R}$ & 27 & 170 & 122 & Total $=319$ \\
\hline BLR recession & 1 & 8 & 1 & Total $=10$ \\
\hline Others & 1 & 2 & 1 & Total $=4$ \\
\hline
\end{tabular}

Abbreviations: BLR recession, both lateral rectus recession; $\mathrm{PD}$, prism dioptre; $\mathrm{R}$ and $\mathrm{R}$, lateral rectus recession and medial rectus recession. ${ }^{*} \mathrm{P}$-value by one-way ANOVA; ${ }^{+} P$-value by Chi-square test.

Group I=IXT with hyperopia; Group II = IXT with emmetropia; Group III = IXT with myopia.

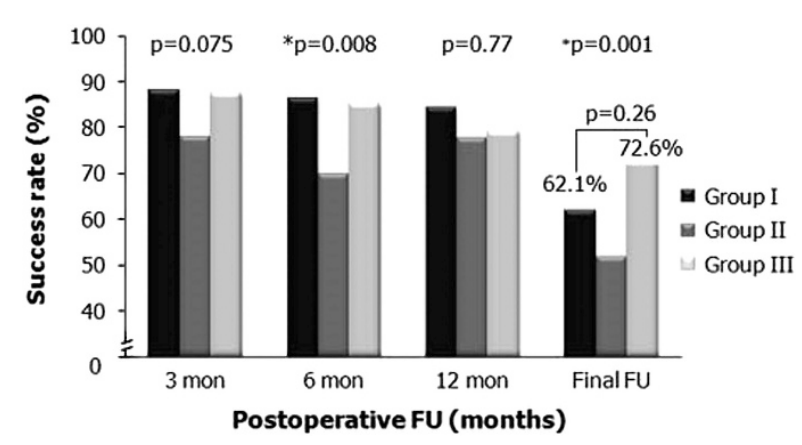

Figure 1 The surgical success rates according to the motor criteria only indicate higher success rates in groups I and III than in group II at postoperative 3, 6, and 12 months and at the last follow-up. At the last follow-up, there was a significant difference in the success rate among the three groups $(P=0.001)$; however, comparisons between group I $(62.1 \%)$ and group III $(72.1 \%)$ demonstrated no significant difference $(P=0.26)$. *Statistically significant by Chi-square test with Bonferroni correction.

undercorrected hyperopic spectacles postoperatively, while only 22 patients of group III (17.7\%) were prescribed spectacles that were more myopic than their own refractive errors $(P<0.001$ by Chi-square test).

\section{Discussion}

This study is a large single surgeon case series assessing the effects of refractive errors following surgery for IXT according to motor and functional criteria. As previously mentioned, success rates of IXT surgery in the literature vary widely from 42 to $81 \%{ }^{4-9}$ Pratt-Johnson et al ${ }^{7}$ explained that when they reported $81 \%$ success in motor alignment, only $41 \%$ of patients had good stereopsis. Pineles et al ${ }^{10}$ reported that, for an excellent motor result in their cohort, the determined success rate was $64 \%$, yet, when considering sensory outcomes, the success rate falls to $38 \%$. In the present study, without reference to groups, the final success rate according to the motor criteria is $60.4 \%$ overall at the final follow-up visit (unpresented data), whereas final success rates according to motor and sensory criteria decreased to $55.1 \%$. Direct comparisons of success rates between different studies are difficult because patient populations, follow-up periods, surgical procedure, and definitions of surgical success differ among the studies; however, our data generally share similar results to previous studies in that functional success rates considering sensory outcome are lower than motor success rates.

In the present study, the surgical success rate was similar in hyperopic and myopic patients and their success rates were better than those with emmetropia regardless of inclusion of functional criteria for successful surgery. We hypothesize that hyperopic refractive error is a good prognostic factor because of the following three reasons. First, myopic patients in this study were significantly older than the other groups at the time of IXT surgery. Lim $e a^{23}$ reported that increased patient age at 
Table 2 Comparisons of preoperative and postoperative stereopsis (log arcsec)

\begin{tabular}{lcccc}
\hline & Group I $(\mathrm{N}=29)$ & Group II $(\mathrm{N}=180)$ & Group III $(\mathrm{N}=124)$ & P-value* \\
\hline Preoperative & $2.29 \pm 0.58$ & $2.03 \pm 0.41$ & $1.92 \pm 0.34$ & $0.002^{+}$ \\
3 months (log arcsec) & $1.99 \pm 0.29$ & $1.92 \pm 0.31$ & $1.85 \pm 0.24$ & 0.130 \\
months (log arcsec) & $1.87 \pm 0.22$ & $1.88 \pm 0.24$ & $1.85 \pm 0.22$ & 0.813 \\
12 months (log arcsec) & $1.89 \pm 0.28$ & $1.84 \pm 0.28$ & $1.71 \pm 0.17$ & 0.055 \\
Final follow-up (log arcsec) & $1.89 \pm 0.24$ & $1.82 \pm 0.26$ & $1.83 \pm 0.26$ & 0.012 \\
\hline
\end{tabular}

$P$-value $=0.007$ by repeated-measures analysis of variance, ${ }^{*} P$-values by one-way analysis of variance; ${ }^{\dagger} P<0.05$.

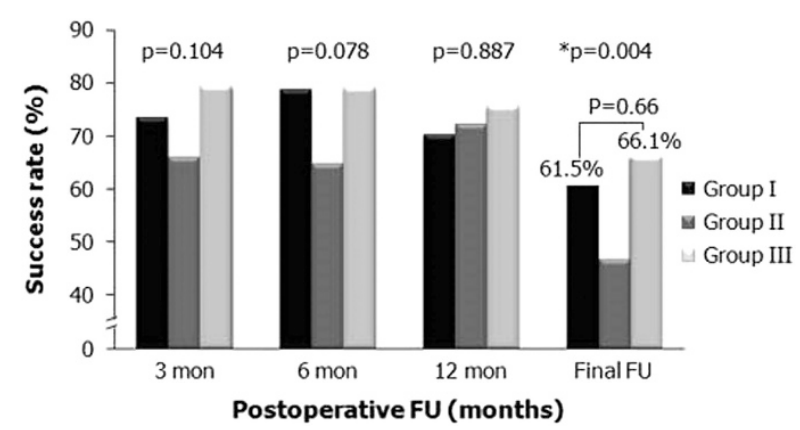

Figure 2 The surgical success rates according to motor and sensory criteria both indicate higher success rates in groups I and III than in group II at postoperative 3 and 6 months and at the last follow-up. There was a statistically significant difference in the success rates between the three groups at the last follow-up $(P=0.004)$; however, the difference between group I $(61.5 \%)$ and group III $(66.1 \%)$ was not significant $(P=0.066)$. *Statistically significant by Chi-square test with Bonferroni correction.

surgery was associated with good prognosis. In older patients, accurate measurements of the angle of deviation are possible, thus allowing more accurate determination regarding the amount of surgery required. ${ }^{24,25}$ Burke $^{26}$ reported that most children with exotropia have periods of normal alignment, during which binocular cooperation can develop normally, so older patients have better fusional ability. Therefore, older myopic patients may have better surgical success rates; however, they demonstrated similar results to hyperopic patients despite their potentially favorable age in our study. Second, although not statistically significant, the follow-up period was longer in hyperopic patients than in other patients. It is known that, in most cases, exotropic drift occurs after IXT surgery, and IXT recurrence rate increases with longer duration of follow-up. 6,23,27 Longer follow-up periods in the hyperopic group could have relatively worse surgical prognoses, yet their results are similar to those of myopic patients. Third, preoperative stereopsis was worse in hyperopic patients than in myopic or emmetropic patients, and there was no difference with respect to stereopsis between the three groups after surgery. This may imply a larger improvement of stereopsis in hyperopic patients. Considering the age effect, follow-up period after IXT surgery, and stereopsis improvement, hyperopic refractive error may be a good prognostic factor for IXT surgery.

Several studies have investigated factors that possibly affect surgical outcomes, including refractive errors; however, there is no consensus regarding the association between IXT surgical results and refractive errors. Koklanis and Georglevski ${ }^{28}$ and Lim et al ${ }^{23}$ found that refractive error was not associated with IXT surgical prognosis. On the other hand, Gezer et al ${ }^{25}$ suggested that patients with higher myopic refractive errors respond less favorably to the same amount of surgery than patients with emmetropia or hypertropia. They explained this relationship by suggesting that myopic patients' measured deviation was slightly larger than their true angle of deviation owing to the effect of minus lenses that increase the measured deviation of strabismus, therefore more of these patients tended to overcorrect. ${ }^{29}$ Some reports explained the relationship between refractive error and surgical response by axial length. ${ }^{30,31}$ For instance, small variations in the radius of the eyeball can have a significant effect on the amount of surgery needed to correct horizontal strabismus. In other words, to correct IXT for eyes with longer axial lengths, surgeons should increase the amount of surgery and vice versa. However, studies report no clinically significant differences in IXT surgical prognosis according to axial length. ${ }^{32,33}$

Hyperopic correction of IXT patients is generally expected to increase the amount of exodeviation by decreasing the demand on accommodative convergence, ${ }^{20}$ however, it may decrease the amount of exodeviation. Iacobucci et $a l^{21}$ and Kushner ${ }^{22}$ report that some exotropic patients with hyperopia paradoxically experience a reduction of exotropia after spectacle correction. These patients had either moderate hyperopia with presumably low AC/A ratio or severe hyperopia, and the authors suggested that, without hyperopia correction, patients may have exerted insufficient accommodative effort because they are unable to sustain the amount of accommodation necessary for a clear image. With spectacle correction, their fusional convergence may facilitate the resolution of exotropia. Chung et $a^{34}$ reported that, in patients with hyperopia, exodeviation increased significantly after optical 
correction in many patients by more than $10 \mathrm{PD}$ at both near and distance. In their report, the increasing exotropia with spectacles was more pronounced in patients with IXT who had pure hyperopia than in patients with IXT who had hyperopic astigmatism $(> \pm 1.50 \mathrm{D}$ of astigmatism) or amblyopia. The authors explained that spectacle correction in astigmatism or amblyopia resulted in clearing blurred retinal images and might have improved fusional convergence with a consequent reduction of exotropia, ${ }^{34}$ whereas hyperopic correction in patients without these concomitant conditions reduces the need for accommodative convergence and thus increases exodeviation. In our study, no patient in group I had $>5.00 \mathrm{D}$ of hyperopia and the mean $\mathrm{AC} / \mathrm{A}$ ratio was within the normal range. Furthermore, amblyopic patients were excluded and $73.68 \%$ of group I had mainly hyperopia (with $< \pm 1.50 \mathrm{D}$ of astigmatism) rather than hyperopic astigmatism. Thus, in our study, hyperopic correction is expected to decrease the demand on accommodative convergence and increase exodeviation, while undercorrection of hyperopic refractive error can do the opposite, ie, decrease the degree of exodeviation. In the present study, $62.1 \%$ of group I patients were prescribed undercorrected hyperopic spectacles after IXT surgery and this might be a contributing factor for better surgical outcome.

This study is a retrospective study and has several limitations. First, there is variability within our patient population. The sample size of the hyperopia group was much smaller than that in the other groups and thus the age and follow-up time ranges are wide, and the age effect could not be properly accounted for. Second, stereopsis was evaluated using only near stereoauity measures (Titmus test) in this study. Distance stereoacuity has been commonly used to monitor deterioration of IXT, because it is known to correlate better with the course of IXT than near stereoacuity. ${ }^{35-37}$ However, Holmes et al ${ }^{38}$ reported that monitoring near stereoacuity is reasonable in children with IXT, because the functional importance of near stereoacuity in children, as well as distant stereoacuity, has been clearly established, and younger children are more interested in near works and functional works such as pegboard task, water task, and bead task, which are related to near stereoacuity. ${ }^{39}$ In our study, most patients were relatively young children, therefore improvement in near stereopsis is an important result. Nonetheless, further studies without these limitations are warranted.

In conclusion, hyperopic refractive error was a good prognostic factor in IXT surgery in this patient population. Furthermore, postoperative undercorrection of hyperopic refractive error might be a contributing factor to surgical outcome in hyperopic IXT.

\section{Summary}

\section{What was known before}

- Several studies have investigated factors that possibly affect surgical outcomes, including refractive errors. The effects of refractive errors in the management of IXT are reported as variable, especially with respect to hyperopia.

What this study adds

- In the present study, the surgical success rate was similar in hyperopic and myopic patients and their success rates were better than those with emmetropia, whether the functional criteria for successful surgery was included or not.

- In the surgical treatment of IXT, taking into consideration the age effect, follow-up period after IXT surgery, and stereopsis improvement, hyperopic refractive error is rather a good prognostic factor.

\section{Conflict of interest}

The authors declare no conflict of interest.

\section{Author contributions}

Study design: MKK, S-HB; data collection: MKK, S-HB; data analysis: MKK, USK, M-JC, S-HB; and manuscript drafting and revision: MKK, S-HB.

\section{Acknowledgements}

This study was supported by Kim's Eye Hospital Research Centre.

\section{References}

1 Friedmann Z, Neumann E, Hyams SW, Peleg B. Ophthalmic screening of 38,000 children, age 1 to $21 / 2$ years, in child welfare clinics. J Pediatr Ophthalmol Strabismus 1980; 17: 261-7.

2 Nusz KJ, Mohney BG, Diehl NN. The course of intermittent exotropia in a population-based cohort. Ophthalmology 2006; 113: 1154-1158.

3 Preslan MW, Novak A. Baltimore vision screening project. Ophthalmology 1996; 103: 105-109.

4 Clarke WN, Noel LP. Surgical results in intermittent exotropia. Can J Ophthalmol 1981; 16: 66-69.

5 Ekdawi NS, Nusz KJ, Diehl NN, Mohney BG. Postoperative outcomes in children with intermittent exotropia from a population-based cohort. J AAPOS 2009; 13: 4-7.

6 Hardesty HH, Boynton JR, Keenan JP. Treatment of intermittent exotropia. Arch Ophthalmol 1978; 96: 268-274.

7 Pratt-Johnson JA, Barlow JM, Tillson G. Early surgery in intermittent exotropia. Am J Ophthalmol 1977; 84: 689-694.

8 Richard JM, Parks MM. Intermittent exotropia: surgical results in different age groups. Ophthalmology 1983; 90: $1172-1177$.

9 Weinstein GS, Biglan AW, Hiles DA. Postoperative residual small angle exodeviations. Ophthalmic Surg 1982; 13: 478-482. 
10 Pineles SL, Ela-Dalman N, Zvansky AG, Yu F, Rosenbaum AL. Long-term results of the surgical management of intermittent exotropia. J AAPOS 2010; 14: 298-304.

11 Beneish R, Flanders M. The role of stereopsis and early postoperative alignment in long-term surgical results of intermittent exotropia. Can J Ophthalmol 1994; 29: 119-124.

12 Ko BW, Shin SY. The clinical features of patients with early recurrence and with orthophoria after intermittent exotropia surgery. J Korean Ophthalmol Soc 2008; 49(7): 1108-1113.

13 Kim SJ, Choi DG. The clinical features in patients with orthophoria over three years after surgery of exotropia. J Korean Ophthalmol Soc 2006; 47(12): 1999-2004.

14 Kim JH, Kim HJ, Choi DG. Contralateral lateral rectus recession versus recess-resect for recurrent exotropia after unilateral recess-resect. Br J Ophthalmol 2013; 97: 752-756.

15 Jang JH, Park JM, Lee SJ. Factors predisposing to consecutive esotropia after surgery to correct intermittent exotropia. Graefes Arch Clin Exp Ophthalmol 2012; 250: 1485-1490.

16 Yam JC, Wu PK, Chong GS, Wong US, Chan CW, Ko ST. Long-term ocular alignment after bilateral lateral rectus recession in children with infantile and intermittent exotropia. J AAPOS 2012; 16: 274-279.

17 Suh SY, Kim MJ, Choi J, Kim SJ. Outcomes of surgery in children with early-onset exotropia. Eye 2013; 27: 836-840.

18 Pineless SL, Deltz LW, Velez FG. Postoperative outcomes of patients initially overcorrected for intermittent exotropia. J AAPOS 2011; 15: 527-531.

19 Buck D, Powell CJ, Rahi J, Cumberland P, Tiffin P, Taylor R et al. The improving outcomes in intermittent exotropia study: outcomes at 2 years after diagnosis in an observational cohort. BMC Ophthalmol 2012; 12: 1.

20 Von Noorden GK. Exodeviations Binocular Vision and Ocular Motility, 6th ed. Mosby: St Louis, USA, 2002, pp 365-366.

21 Iacobucci IL, Archer SM, Giles CL. Children with exotropia responsive to spectacle correction of hyperopia. Am J Ophthalmol. 1993; 116: 79-83.

22 Kushner BJ. Surgical pearls for the management of intermittent exotropia. Am Orthopt J 1992; 42: 65-71.

23 Lim SH, Hwang BS, Kim MM. Prognostic factors for recurrence after bilateral rectus recession procedure in patients with intermittent exotropia. Eye 2012; 26: 846-852.

24 Kushner BJ. Selective surgery for intermittent exotropia based on distance/near differences. Arch Ophthalmol 1998; 116: 324-328.

25 Gezer A, Sezen F, Nasri N, Gozum N. Factors influencing the outcome of strabismus surgery in patients with exotropia. J AAPOS 2004; 8: 56-60.
26 Burke MJ. Intermittent exotropia. Int Ophthalmol Clin 1985; 25: 53-68.

27 Scott WE, Keech R, Mash AJ. The postoperative results and stability of exodeviations. Arch Ophthalmol 1981; 99: 1814-1818.

28 Koklanis K, Georglevski Z. Recurrence of intermittent exotropia: factors associated with surgical outcomes. Strabismus 2009; 17: 37-40.

29 Scattergood KD, Brown MH, Guyton DL. Artifacts introduced by spectacle lenses in the measurement of strabismic deviations. Am J Ophthalmol 1983; 96: 439-448.

30 Vazquez RL. The effect of surgical technique and the radius of the eye on correction for horizontal strabismus. Ann Ophthalmol 1987; 19: 187-193.

31 Kushner BJ, Lucchese NJ, Morton GV. Variation in axial length and anatomical landmarks in strabismic patients. Ophthalmology 1991; 98: 400-406.

32 Kushner BJ, Lucchese NJ, Morton GV. The influence of axial length on the response to strabismus surgery. Arch Ophthalmol 1989; 107: 1616-1618.

33 Kushner BJ, Fisher MR, Lucchese NJ, Morton GV. Factors influencing response to strabismus surgery. Arch Ophthalmol 1993; 111: 75-79.

34 Chung SA, Kim IS, Kim WK, Lee JB. Changes in exodeviation following hyperopic correction in patients with intermittent exotropia. J Pediatr Ophthalmol Strabismus 2011; 48: $278-284$.

35 Stathacopoulos RA, Rosenbaum AL, Zanoni D, Stager DR, McCall LC, Ziffer AJ et al. Distance stereoacuity. Assessing control in intermittent exotropia. Ophthalmology 1993; 100: 495-500.

36 O'Neal TD, Rosenbaum AL, Stathacopoulos RA. Distance stereo acuity improvement in intermittent exotropic patients following strabismus surgery. J Pediatr Ophthalmol Strabismus. 1995; 32: 353-357.

37 Yildrim C, Mutlu FM, Chen Y, Altinsoy HI. Assessment of central and peripheral fusion and near and distance stereoacuity in intermittent exotropic patients before and after strabismus surgery. Am J Ophthalmol 1999; 128: 222-230.

38 Holmes JM, Leske DA, Hatt SR, Brodsky MC, Mohney BG. Stability of near stereoacuity in childhood intermittent exotropia. J AAPOS 2011; 15: 462-467.

39 O'Connor AR, Birch EE, Anderson S, Draper H, FSOS Research Group. The functional significance of stereopsis. Invest Ophthalmol Vis Sci 2010; 51: 2019-2023. 